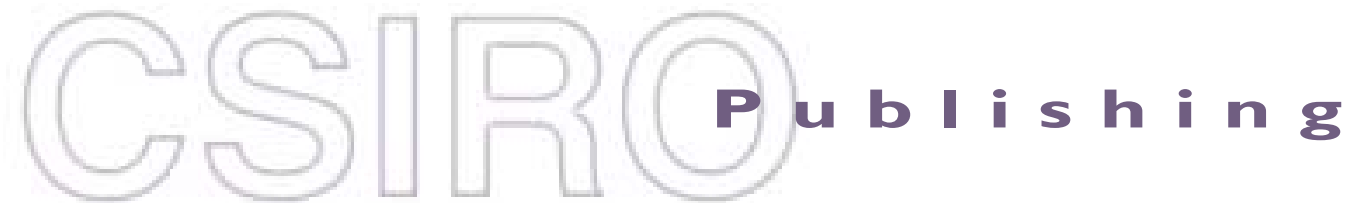

\section{Publications of the Astronomical Society of Australia}

Volume 19, 2002

(C) Astronomical Society of Australia 2002

An international journal of astronomy and astrophysics

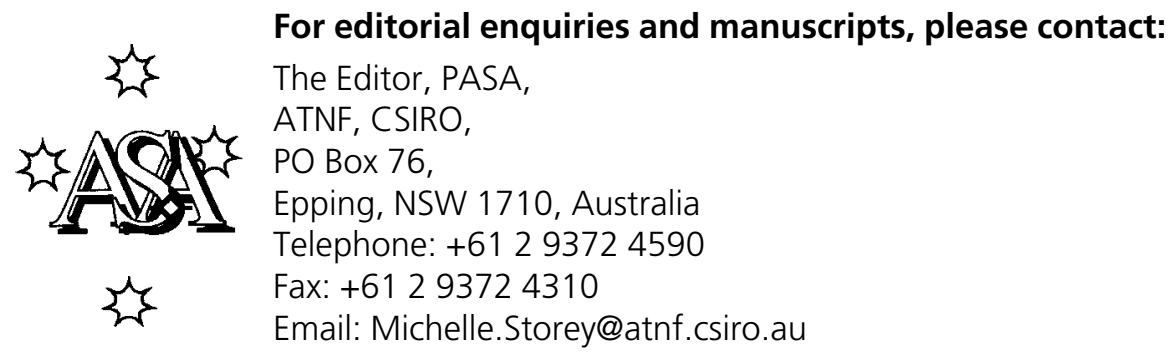

For general enquiries and subscriptions, please contact: CSIRO Publishing PO Box 1139 (150 Oxford St)

Collingwood, Vic. 3066, Australia

Telephone: +6139662 7666

Fax: +61 396627555

Email: publishing.pasa@csiro.au

C S I RO

PUBLISHING Published by CSIRO Publishing

for the Astronomical Society of Australia

www.publish.csiro.au/journals/pasa 


\title{
Broad-band Radio Spectra Variability of 550 AGN in 1997-2001
}

\author{
Y. Y. Kovalev ${ }^{1}$, Yu. A. Kovalev ${ }^{1}$, N. A. Nizhelsky ${ }^{2}$ and A. B. Bogdantsov ${ }^{2}$ \\ ${ }^{1}$ Astro Space Center of P. N. Lebedev Physical Institute, Profsoyuznaya 84/32, \\ Moscow, 117997 Russia \\ yyk@asc.rssi.ru \\ ${ }^{2}$ Special Astrophysical Observatory, Nizhny Arkhyz, KChR, 357147 Russia \\ Received 2001 August 4, accepted 2002 February 13
}

\begin{abstract}
Results are presented of long-term monitoring at the radio telescope RATAN-600 and a model interpretation of instantaneous 1-22 GHz spectra at six frequencies for a sample of 550 compact extragalactic radio sources. The sources are selected from the Preston et al. (1985) VLBI survey and are located in the declination range from $-30^{\circ}$ to $+43^{\circ}$. Dependence of the index of variability on frequency is analysed for different subsamples. Factors of flux density variations range from 1 to 15 . A typical behaviour of spectra evolution during strong radio outbursts in various objects is recognised suggesting a common physical nature of the variability. Invoking VLBI observations in spectra-structure analysis, we find that usually more than $70 \%$ of the total emission is coming from the mas scale at frequencies higher than $5 \mathrm{GHz}$. The observed variability can be explained in the model with a relativistic jet of parsec scale in the longitudinal magnetic field or in the shock-in-jet model.
\end{abstract}

Keywords: galaxies: active — galaxies: jets — BL Lacertae objects: general — quasars: general — radio continuum: galaxies

\section{Introduction}

The physical processes causing the variability of AGN emission have long been of great interest. Much attention has been paid to sources with high amplitude flares, especially if a flare occurs after a quiescent stage. In such a case subsequent flares do not overlap (as frequently happens), and the observed flux is most likely the sum of a quiescent and an evolving powerful component emission. This situation is very suitable for comparison with predictions of theoretical models as there is no need to involve a large number of free parameters. By discovering the general properties of flares for different optical classes of AGN, we can conclude how strong are the differences between them.

\section{Observations}

Observations were made from 1997 March to 2001 September (12 epochs), at $31,13,7.7,3.9,2.7$, and $1.4 \mathrm{~cm}$, simultaneously for all frequencies, with the $600-\mathrm{m}$ ring radio telescope RATAN-600 in the framework of a longterm monitoring program. In 1997 March we started the program for a sample of about 550 compact extragalactic sources with correlated flux density $F_{c}>0.1 \mathrm{Jy}$ at $13 \mathrm{~cm}$ from the Preston et al. (1985) VLBI survey in the declination range from $-30^{\circ}$ to $+43^{\circ}$. Results of the observations made in 1997 December, the list of the sources included in the monitoring, description of the method of observations and data processing are presented in Kovalev et al. (1999). Horizontal localisation of the stationary antenna horns allows broad-band spectral responses to be obtained at all observed frequencies in a time of 1-2 min in the transit mode of meridional observations.

\section{Discussion}

\subsection{Two Main Components of Radio Spectra}

From statistical and numerical model analysis of 550 observed spectra we conclude that the spectra can be modelled as the sum of a synchrotron spectrum of an extended optically thin component (magnetised envelope/ lobe, 'spectrum 1'), dominating at lower frequencies, and a synchrotron spectrum of a compact component (relativistic jet, 'spectrum 2'), dominating at higher frequencies. Spectrum 1 is generally constant or weakly variable, spectrum 2 can exhibit any degree of variability.

In Figure 1 we present examples of fitting the sum of spectra 1 and 2 (with different contributions to the total emission) to the observational data from Kovalev et al. (1999). The data at 5 and $2.7 \mathrm{GHz}$ (for $0312+10$ ), $1.4 \mathrm{GHz}$, and below $1 \mathrm{GHz}$ are taken from the CATS database (Verkhodanov et al. 1997). The model of a quasi-stationary jet in the longitudinal magnetic field is adopted for spectrum 2 (Nesterov et al. 1994). For the quasar 0552+39, the contribution of spectrum 1 is small or even negligible; next are the quasar $0923+39$, the quasar $0838+13$, and the galaxy $0312+10$, successively by increasing the contribution of spectrum 1 .

Considering non-stationary jets, a combination of two spectral components can explain the features of spectra with complex shapes. A variable emission of a jet can be produced by the non-stationary outflow of relativistic particles in the longitudinal magnetic field (see e.g. Kovalev, Kovalev, \& Nizhelsky 2000) or by the propagation of relativistic shocks (see e.g. Marscher \& Gear 1985).

We have compared the integrated flux, measured in the large VLBA survey programs (Fey \& Charlot 1997; 


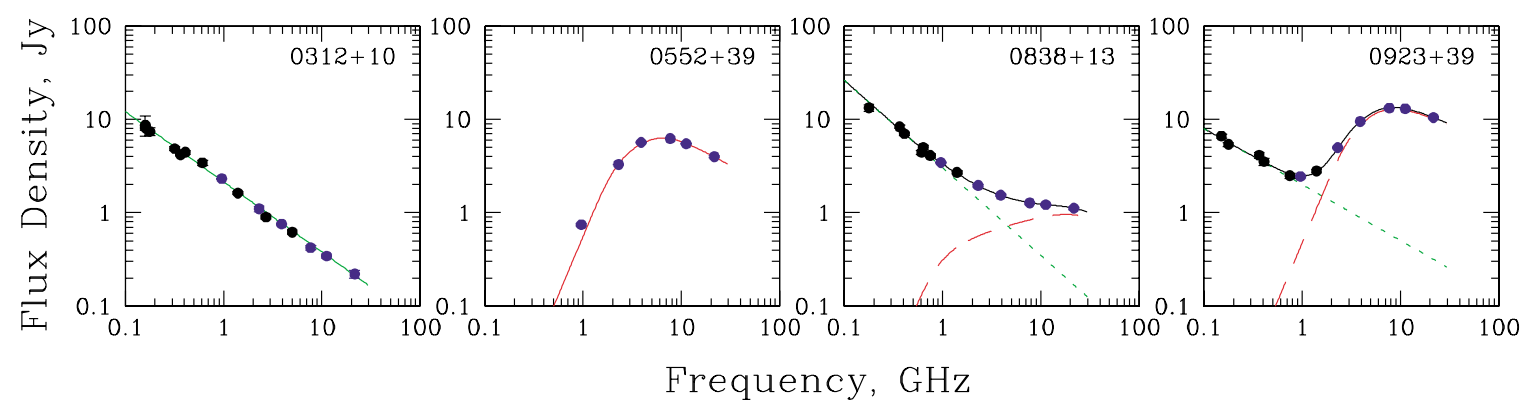

Figure 1 Examples of the model spectral fits. Total spectrum is the sum of a spectrum of an extended optically thin component (magnetised envelope/lobe), constant or weakly variable and dominating at lower frequencies, and a spectrum of a compact component (relativistic jet), dominating at higher frequencies, with any degree of variability. Errors are smaller than the point size when they are not visible.

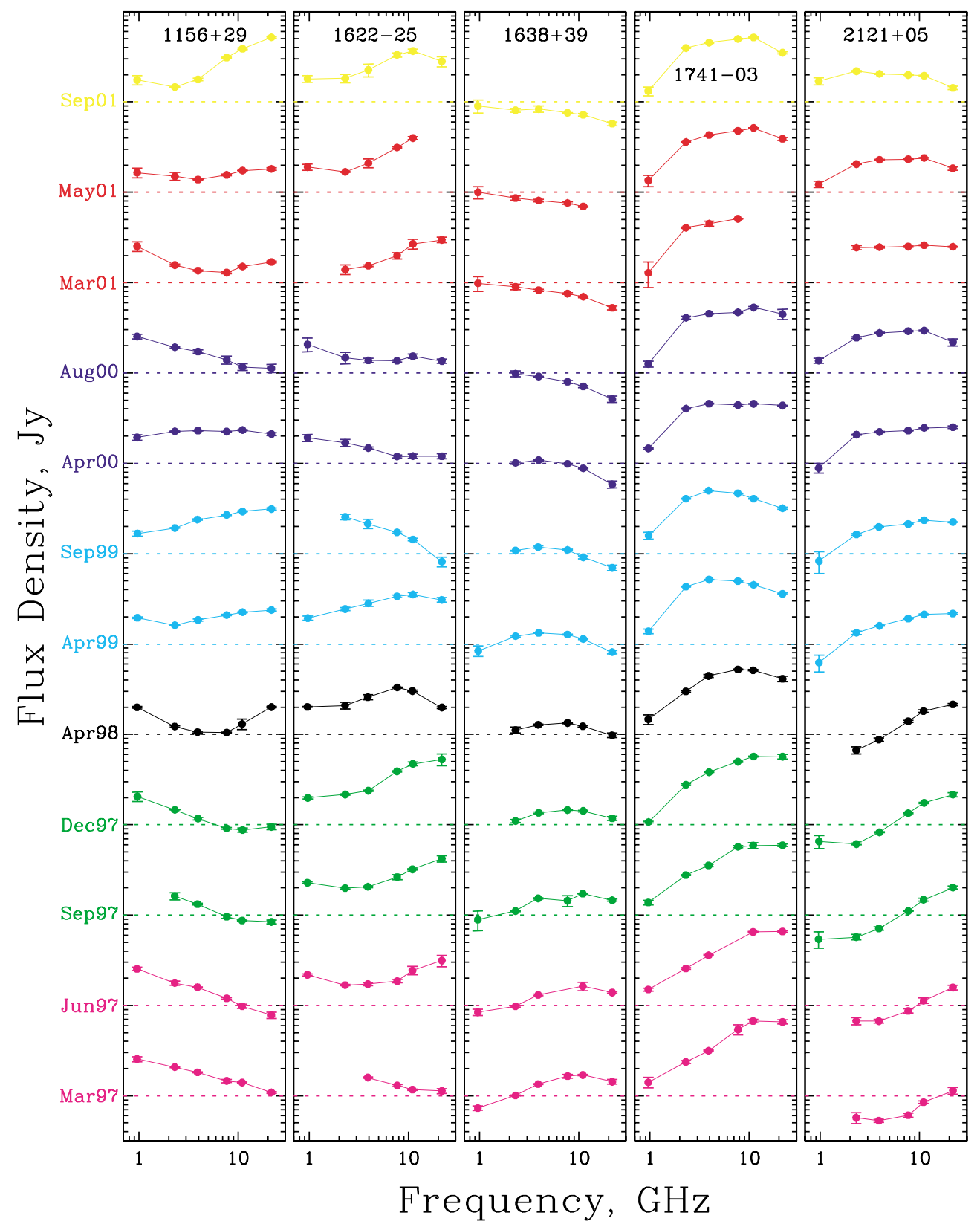

Figure 2a Examples of strong long-term variability of broad-band instantaneous spectra in 1997-2001. Evolution of the spectra is shown. Spectra for different epochs are given along the height of the boxes. Dotted horizontal lines mark 1 Jy levels for each epoch. The spectra for these sources among others are presented in Figure $2 \mathrm{~b}$ without shifting. The error is smaller than the point size when an error bar is not visible. 

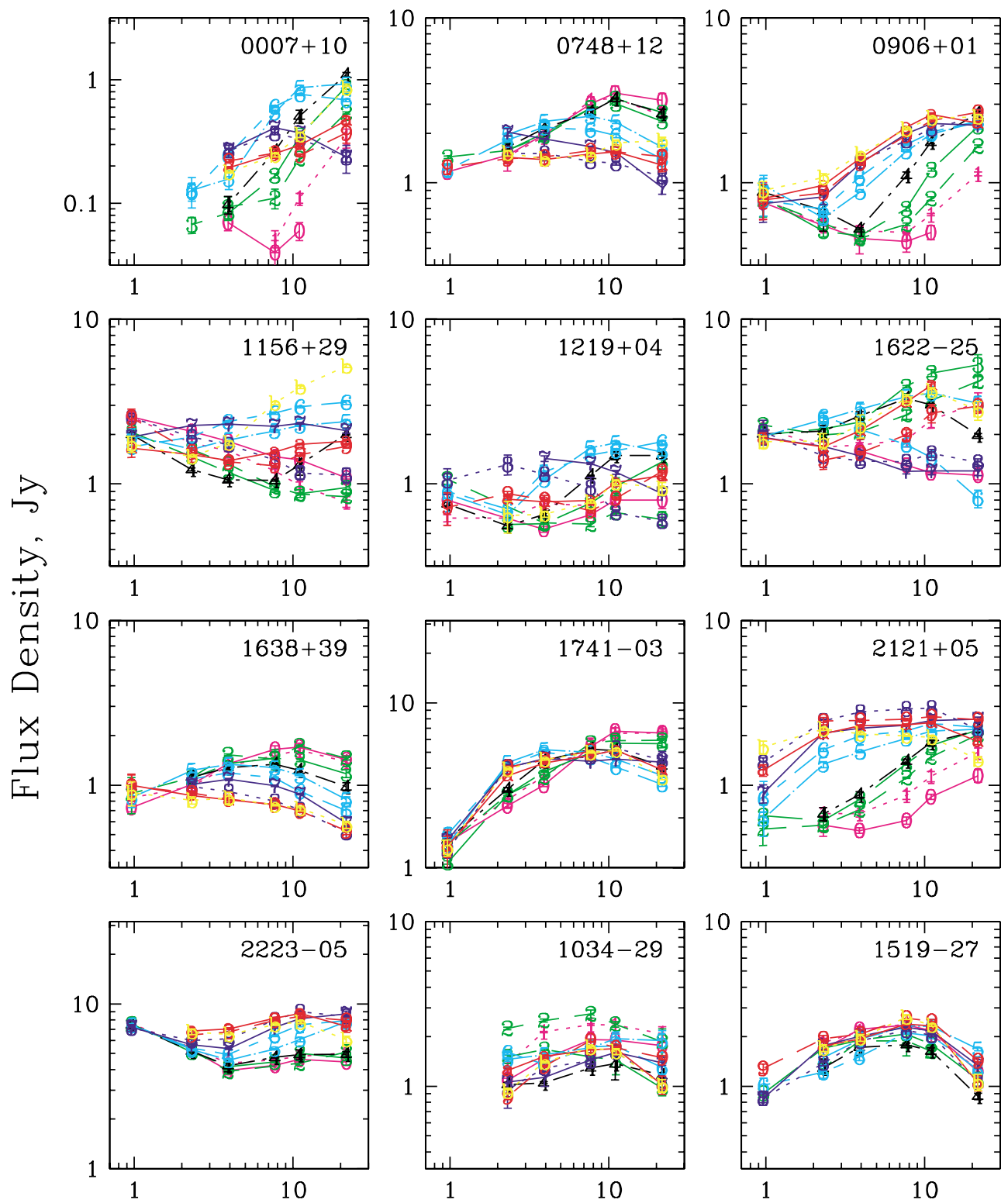

Frequency, GHz

Figure 2b Examples of strong long-term variability of broad-band instantaneous spectra in 1997-2001. Shown are the multi-epoch 1-22 GHz spectra for epochs 1997 March, June, September, December; 1998 March; 1999 April, September; 2000 April, August; 2001 March, May, September (12 epochs, labelled ' 0 ' through ' 9 ' and ' $a$ ', 'b'). The error is smaller than the label size when an error bar is not visible.

Kellermann et al. 1998) and in our EVN+ observations of selected flaring AGN, with the results of our RATAN-600 observations. From the comparison we conclude that usually more than $70 \%$ of the total emission is coming from the mas scale at frequencies higher than $5 \mathrm{GHz}$. This result confirms our previous interpretation of (i) quiescent spectra by the emission of a jet in a quasi-stationary phase, and (ii) the spectra variability at frequencies higher than $1 \mathrm{GHz}$ by the strongly variable spectrum 2 .

\subsection{Radio Flares Evolution}

Onsets of strong radio flares in about 100 AGN were detected in 1997-2001. Examples of spectra variability for some of these sources are presented in Figure 2. A typical behaviour of spectra evolution during strong radio outbursts is recognised: a wave of a perturbation moves along the spectrum from higher to lower frequencies (Figure 2). Quasars and BL Lacertae objects have a similar type and rate of long-term variability. Therefore, the hypothesis of the same physical nature of quasars and BL Lacs is supported.

Objects of dissimilar type of variability were also observed. Spectra of two of them, 1034-29 and 1519-27, are displayed at the bottom of Figure $2 b$. Variability features of these objects are explained by interstellar scintillation (see Macquart et al. 2000, Kedziora-Chudczer et al. 2001). 
Table 1. Mean variability index vs frequency dependence for different subsamples

\begin{tabular}{lcccccr}
\hline Subsample & $\begin{array}{c}\text { Number of } \\
\text { objects }\end{array}$ & \multicolumn{5}{c}{$\nu, \mathrm{GHz}$} \\
\cline { 3 - 7 } & & 22 & 11 & 7.7 & 3.9 & 2.3 \\
\hline$\gamma$-ray bright & 35 & 0.35 & 0.30 & 0.27 & 0.19 & 0.16 \\
HP objects & 78 & 0.32 & 0.28 & 0.25 & 0.20 & 0.17 \\
BL Lacs & 48 & 0.29 & 0.24 & 0.22 & 0.18 & 0.17 \\
Quasars & 379 & 0.24 & 0.19 & 0.16 & 0.14 & 0.15 \\
Galaxies and unidentified RS & 118 & 0.20 & 0.17 & 0.14 & 0.12 & 0.13 \\
\hline
\end{tabular}

We found a very good agreement between outburst observational data and the model describing synchrotron radiation of a relativistic jet in the longitudinal magnetic field (see results of numerical simulation of spectral variability in Kovalev et al. 2000). In the model an outburst is explained by an increase of the flow of relativistic particles injected from the core region to the jet. An outburst evolution over all frequencies and the decrease of the optical depth at high frequencies are interpreted as the evolution of a new jet component and its motion away from the core. The delayed increase of the flux density at the lower frequencies follows from the model.

We considered the flare evolution also in the frequently suggested model of a shock wave passing through a conical adiabatically expanding jet (Marscher \& Gear 1985). Our analysis shows that the evolution of spectral parameters in time during the flare in most of the flaring objects observed is in qualitative agreement with the shock-in-jet model predictions.

\subsection{Variability Index}

We derived the variability index (relative amplitude of the variability) $V_{v}$ at five frequencies (see Table 1) for different subsamples. The index was calculated for 550 objects over a 4.5-yr period (1997 March-2001 September: 12 epochs) according to the simple form $V_{v}=\left(F_{v, \max }-\right.$ $\left.F_{v, \text { min }}\right) /\left(F_{v, \text { max }}+F_{v, \text { min }}\right)$. More than $20 \%$ of the sources have strong variability at the higher frequencies during this period, with $1.5-15$ times change in the flux (variability index is more than 0.25 ). The mean variability index (Table 1) decreases with decreasing frequency, which is in accordance with predictions of the models mentioned above.

Kovalev et al. (2002) show that highly polarised (HP, with degree of optical polarisation more than 3\%) and BL Lac objects in the sample have, on average, a greater contribution of compact jet emission to the total spectrum than the quasars do. Generally compact jet emission is variable. Thus we expect HP and BL Lac objects to be more variable than the quasars in the sample, which is what we found.

The subsample of the $\gamma$-ray bright objects (detected by EGRET, see Mattox et al. 1997; Mattox, Hartman, \& Reimer 2001) evidently has the maximum value of the variability index at the higher frequencies. This fact confirms previous results (see e.g. Valtaoja \& Teräsranta 1996) and favours the models suggesting a relation between the emission mechanisms in radio and $\gamma$-ray ranges.

\section{Summary}

We claim that the total spectra of AGN can be represented as the sum of two main broad-band spectral components and that the investigated sample consists of compact sources with continuous relativistic jets, with one general physical picture underlying all sources. For different types of AGN the same behaviour of flares is revealed, suggesting a common physical nature of BL Lacs and quasars. The model with the longitudinal magnetic field and the shockin-jet model explain satisfactorily the observed evolution of strong radio flares. The revealed special radio features of the EGRET subsample favours the models suggesting a relation between the emission mechanisms in radio and $\gamma$-ray ranges.

\section{Acknowledgments}

We thank the two anonymous referees for their careful reading of the manuscript and suggestions which have greatly improved the paper. YYK is thankful to the LOC of the AGN Variability Meeting for the local support. This work has been partly supported by the NASA JURRISS program (project W-19611), the Russian Foundation for Basic Research (projects 99-02-17799, 01-02-16812, 01-02-06084), and the Russian State Program 'Astronomy' (project 1.2.5.1). We have made use of the database CATS (Verkhodanov et al. 1997) of the Special Astrophysical Observatory. The European VLBI Network is a joint facility of European and Chinese radio astronomy institutes funded by their national research councils.

\section{References}

Fey, A. L., \& Charlot, P. 1997, ApJS, 111, 95

Kedziora-Chudczer, L. L., Jauncey, D. L., Wieringa, M. H., Tzioumis, A. K., Reynolds, J. E. 2001, MNRAS, 325, 1411

Kellermann, K. I., Vermeulen, R. C., Zensus, J. A., \& Cohen, M. H. 1998, AJ, 115, 1295

Kovalev, Y. Y., Nizhelsky, N. A., Kovalev, Yu. A., Berlin, A. B., Zhekanis, G. V., Mingaliev, M. G., \& Bogdantsov, A. V. 1999, A\&AS, 139, 545 
Kovalev, Yu. A., Kovalev, Y. Y., \& Nizhelsky, N. A. 2000, PASJ, 52,1027

Kovalev, Y. Y., Nizhelsky, N. A., Kovalev, Yu. A., Zhekanis, G. V., \& Bogdantsov, A. V. 2002, ASP Conf. Ser., IAU Col 184, in press

Macquart, J.-P., Kedziora-Chudczer, L., Rayner, D. P., \& Jauncey, D. L. 2000, ApJ, 538, 623

Marscher, A. P., \& Gear, W. K. 1985, ApJ, 298, 114

Mattox, J. R., Hartman, R. C., \& Reimer, O. 2001, ApJ, 135, 155

Mattox, J. R., Schachter, J., Molnar, L., Hartman, R. C., \& Patnaik, A. R. 1997, ApJ, 481, 95
Nesterov, N. S., Kovalev, Y. Y., Babak, S. V., \& Larionov, G. M. 1994, ARep, 38, 757

Preston, R. A., Morabito, D. D., Williams, J. G., Faulkner, J., Jauncey, D. L., \& Nicolson, G. D. 1985, AJ, 90, 1599

Valtaoja, E., \& Teräsranta, H. 1996, A\&AS, 120, 491

Verkhodanov, O. V., Trushkin, S. A., Andernach, H., \& Chernenkov, V. N. 1997, in Astronomical Data Analysis Software and Systems VI, ASP Conf. Ser. 125, eds G. Hunt, \& H. E. Payne (San Francisco: ASP), 322 